\title{
Interferon $\beta$ treatment for multiple sclerosis has a graduated effect on MRI enhancing lesions according to their size and pathology
}

\author{
Neuroimaging \\ Research Unit \\ M Filippi \\ $M$ Rovaris \\ M P Sormani

\begin{abstract}
Clinical Trials Unit, Department of

Neuroscience, Scientific Institute Ospedale San Raffaele, University of Milan, Milan, Italy V Martinelli

G Comi
\end{abstract}

Department of

Neurology

R Capra

Department of
Neuroradiology,
Spedali Civili,
University of Brescia,
Brescia, Italy
F Prandini

Department of

Neurology

C Gasperini

C Pozzilli

Department of Neuroradiology,

Università "La

Sapienza", Rome, Italy

S Bastianello

Division of Medical Physics, University of Leicester, Leicester, UK

M A Horsfield

Unit of Clinical Epidemiology and Trials, National Institute for Cancer Research, Genoa, Italy M P Sormani

Correspondence to: Dr Massimo Filippi, Neuroimaging Research Unit, Department of Neuroscience, Scientific Institute Ospedale San Raffaele, Via Olgettina 60, 20132 Milan, Italy. Telephone 0039022643 3033; fax 0039022643 3031

Received 27 October 1998 and in revised form 4 February 1999

Accepted 18 March 1999

\begin{abstract}
M Filippi, M Rovaris, R Capra, C Gasperini, F Prandini, V Martinelli, M A Horsfield,
\end{abstract} S Bastianello, M P Sormani, C Pozzilli, G Comi

tive-The ability of recombinant interferon $\beta-1 \mathrm{a}$ (rh-IFN $\beta$-1a) to -Every 4 weeks, standard dose (Sd; $0.1 \mathrm{mmol} / \mathrm{kg} \mathrm{Gd}$ ) and triple dose (Td; $0.3 \mathrm{mmol} / \mathrm{kgGd}$ ) MRI were obtained from 18 patients with relapsing-remitting multiple sclerosis for 3 months before and 4 months after starting treatment with $44 \mu \mathrm{g}$ rh-IFN $\beta$-1a subcutaneously, once a week. Results-The total numbers of enhancing lesions were 145 and 126 on Sd scans and 278 and 192 on the Td scans obtained before and after treatment. The introduction of treatment decreased, on average, the rate of appearance of new enhancing lesions seen on $\mathrm{Sd}$ and $\mathrm{Td}$ scans by $37 \%$ $(p<0.001)$. Treatment effects on new enhancing lesions seen on Td scans was, on average, $28 \%$ higher than on those seen on Sd scans. The distribution of lesion sizes on Td scans changed significantly during the treatment period $(p=0.05)$, due to a marked decrease in the number of small lesions. Conclusions - The effect of $44 \mu \mathrm{g}$ rh-IFN $\beta-1 a$ in reducing multiple sclerosis disease activity, as monitored by Gd enhanced MRI, is not homogeneous, but graduated according to the pathological characteristics and size of the lesions.

(f Neurol Neurosurg Psychiatry 1999;67:386-389)

Keywords: multiple sclerosis; interferon $\beta$; magnetic resonance imaging

In patients with relapsing-remitting multiple sclerosis, treatment with interferon (IFN) $\beta-1 b^{1-3}$ or IFN $\beta-1 a^{4-7}$ results in a significant reduction in the number of active multiple sclerosis lesions, as measured by the frequency of enhancement on serial T1 weighted MRI with a standard dose (Sd) of gadoliniumDTPA (Gd) contrast agent. However, recent studies have shown that the yield of active mul-

tiple sclerosis lesions can be increased using several strategies. ${ }^{8-10}$ Of these, the use of a triple dose (Td) of contrast agent is by far the most effective. ${ }^{9} 10$

Lesions which are only seen to enhance after injecting a $\mathrm{Td}$ of $\mathrm{Gd}$ form a large subgroup of the population of active multiple sclerosis lesions, and are thought to represent those with a low grade inflammation. ${ }^{11}$ These lesions have indeed a less damaged blood-brain barrier and a less severe tissue disorganisation, as measured by magnetisation transfer ratio (MT ratio) changes, at the time of their formation and during a 3 month follow up compared with those enhancing on Sd scans. ${ }^{11}$

The high sensitivity of Td enhanced MRI to multiple sclerosis activity may result in earlier detection of a treatment effect and a reduction in the number of scans needed to show it. ${ }^{10} \mathrm{On}$ the other hand, the magnitude of the effect of IFN $\beta$ on lesions enhancing at different $\mathrm{Gd}$ doses may give further information about the mode of action of the drug in multiple sclerosis. In the present study, we evaluated the ability of $44 \mu \mathrm{g}$ recombinant human IFN $\beta$-1a (rh-IFN $\beta-1$ a) administered once a week to suppress multiple sclerosis activity, measured across a range of enhancing lesion types.

\section{Patients and methods}

PATIENTS

Eighteen patients (nine women and nine men) 
after treatment initiation) were treated with intravenous methylprednisolone ( $1 \mathrm{~g} /$ day for 3 days).

STUDY DESIGN

A baseline versus treatment trial design was used. Brain MRI was performed every 28 ( \pm 5$)$ days on eight separate occasions, and treatment with rh-IFN $\beta-1$ a was initiated the day after the fourth MRI session. In cases of steroid treatment, MRI was always scheduled either before the start of the treatment or 10 days after the end. No other immunosuppressive or immunomodulating treatment was allowed during the study.

TREATMENT

Treatment consisted of administration of $44 \mu \mathrm{g}$ rh-IFN $\beta$-1a (Rebif ${ }^{\circledR}$, Ares-Serono, Geneva, Switzerland) subcutaneously, once a week.

MAGNETIC RESONANCE IMAGING

Scanners operating at 1.5 Tesla were used at all three centres. On each scanning occasion, the MRI examination was split into two sessions, separated by an interval of between 12 and 24 hours. During the first session, the following scans were performed: (a) dual echo conventional spin echo (CSE) $(T R=2000-2400$, first echo $\mathrm{TE}=30-50$, second echo $\mathrm{TE}=80-100$, number of acquisitions $=1)$; $(b)$ precontrast $\mathrm{T} 1$ weighted CSE (TR=560-768, TE $=14-15$, number of acquisitions $=2)$; (c) postcontrast $\mathrm{T} 1$ weighted scans, with the same acquisition parameters as before $\mathrm{Gd}$ injection, 5 minutes after the injection of Gd. During the second session, the following were performed with the same parameters as above: (a) precontrast T1 weighted scan; $(b)$ postcontrast $\mathrm{T} 1$ weighted scans 5 minutes after the $\mathrm{Gd}$ injection. The dose of $\mathrm{Gd}$ was randomised so that in the first session the patient received either an $\mathrm{Sd}(0.1$ $\mathrm{mmol} / \mathrm{kg})$ or $\mathrm{Td}(0.3 \mathrm{mmol} / \mathrm{kg})$ of $\mathrm{Gd}$, with the opposite dose given during the second session.

For all the scans, 24 contiguous interleaved axial slices were acquired with $5 \mathrm{~mm}$ slice thickness, 192-256×256 raw data matrix, and 220-250 mm square field of view. For follow up scans, the scan planes were carefully repositioned according to published guidelines. ${ }^{15}$

IMAGE REVIEW

The enhanced $\mathrm{T} 1$ weighted scans were assessed to determine the total number of enhancing lesions and the number of new enhancing lesions, in a manner described in previously published studies. ${ }^{10}{ }^{16} \mathrm{~A}$ single technician, unaware of the $\mathrm{Gd}$ dose used, the patient to whom the scans belonged and the time point of the acquisition, measured the volumes of enhancing lesions using a semiautomated local thresholding technique. ${ }^{17}$ After observing the volume distribution of the pooled $\mathrm{Sd}$ and $\mathrm{Td}$ enhancing lesions, the enhancing lesions were classified according to their size into three groups containing equal numbers of lesions: small (in which the volume was smaller than $0.06 \mathrm{ml}$ ), intermediate (volume greater than $0.06 \mathrm{ml}$ but less than or equal to $0.13 \mathrm{ml}$ ), or large (volume greater than $0.13 \mathrm{ml}$ )

STATISTICAL ANALYSIS

The effect of $\mathrm{Gd}$ dose and treatment on the number of new enhancing lesions was evaluated by a Poisson regression model with patients considered as blocks. The distribution of the mean lesion rates on monthly scans for small, intermediate, and large lesions during the baseline and the treatment periods (seen on both $\mathrm{Sd}$ and $\mathrm{Td}$ scans) were compared by analysis of variance (ANOVA).

\section{Results}

The total numbers of enhancing lesions were 145 (39 small, 55 intermediate, and 51 large) on $47 / 72$ Sd scans obtained during the baseline period, 126 (25 small, 57 intermediate, and 44 large) on $46 / 72 \mathrm{Sd}$ scans obtained during the treatment, 278 (123 small, 77 intermediate, and 78 large) on 54/72 Td scans obtained during the baseline period, and 192 (54 small, 64 intermediate, and 74 large) on $48 / 72 \mathrm{Td}$ scans obtained during treatment. One patient had no enhancing lesions on $\mathrm{Sd}$ or $\mathrm{Td}$ scans during the baseline period, whereas this was the case for three patients during the treatment period.

On Sd scans, 78 new enhancing lesions were detected over the 3 month baseline period and 79 over the 4 month treatment period. On Td scans, 132 new enhancing lesions were detected over the 3 month baseline period and 96 over the 4 month treatment period. In the table, the estimated frequencies of appearance of new enhancing lesions in each of the four experimental conditions are presented. The final model of the Poisson regression analysis showed that, during the baseline period, the average number of new enhancing lesions seen on Td scans was $69 \%$ (95\% confidence interval (95\% CI) $28-124 \%$ ) higher $(p=0.002)$ than that estimated for Sd scans. The introduction of the treatment decreased, on average, the rate of appearance of new enhancing lesions seen on Sd and Td scans by 37\% (95\% CI 24-49\%) $(p<0.001)$. However, the model also showed that the dose of Gd used changed the apparent treatment effect: on average a more pronounced effect (mean+28\%; 95\% CI 52 to $+8 \%$ ) was seen on $\mathrm{Td}$ scans compared with $\mathrm{Sd}$ scans. This was confirmed by the evaluation of the treatment effects on the new enhancing lesions rates seen on $\mathrm{Sd}$ (average reduction-24\% (range $-45 \%$ to $+3 \%$ ), $\mathrm{p}=0.09$ ) and $\mathrm{Td}$ (average reduction $-45 \%$ (range $-58 \%$ to $-29 \%$ ), $\mathrm{p}<0.001)$ scans.

Mean (95\% CI) for the new enhancing lesion rate on standard dose and triple dose scans during the baseline and the treatment periods estimated using a Poisson regression model

\begin{tabular}{lll}
\hline & \multicolumn{2}{l}{$\begin{array}{l}\text { Mean number (95\% CI) of new enhancing } \\
\text { lesions/patient/month }\end{array}$} \\
\cline { 2 - 3 } & Sd scans & Td scans \\
\hline Baseline period & $1.44(1.15-1.80)$ & $2.44(2.06-2.90)$ \\
Treatment period & $1.09(0.88-1.37)$ & $1.33(1.09-1.63)$ \\
\hline
\end{tabular}

$\mathrm{Sd}=$ Standard dose; $\mathrm{Td}=$ triple dose. 
Standard dose scans

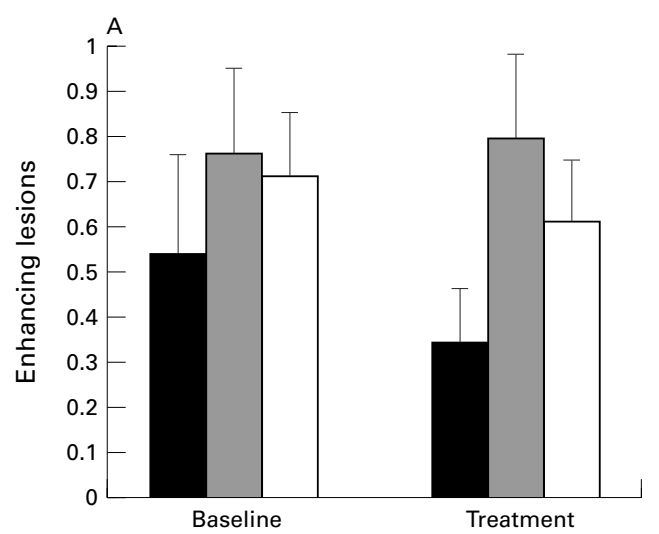

Triple dose scans

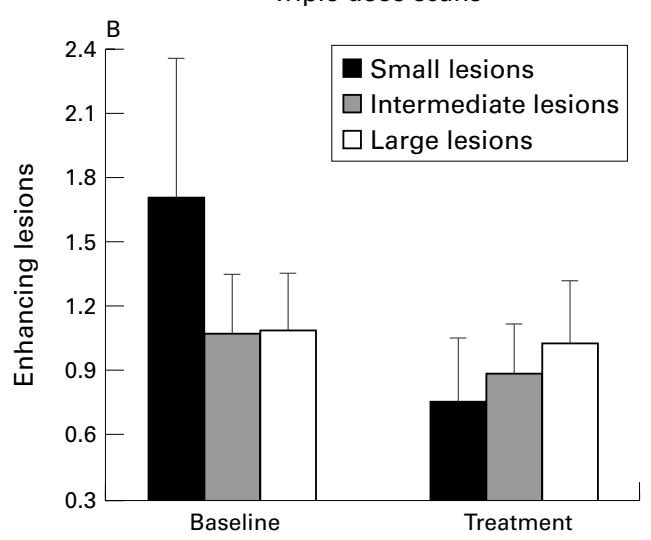

Distribution of enhancing lesion volumes on standard and triple dose scans during the baseline and the treatment periods. The charts show the mean number of enhancing lesions/patient/month with the error bars representing the standard errors.

On Sd scans, the distribution of lesions sizes did not change significantly after treatment $(p=0.70)$, whereas it did on the $\mathrm{Td}$ scans $(p=0.05$, figure). In detail, on $\mathrm{Td}$ scans, the number of small lesions decreased by $56 \%$, intermediate by $17 \%$, and large by only $5 \%$ during the treatment period. Furthermore, when comparing lesion sizes for $\mathrm{Sd}$ and $\mathrm{Td}$, we found that treatment reduced the differences between the two distributions of lesion size. During the baseline period, small lesions were $27 \%$ of the total on Sd and $44 \%$ of the total on $\mathrm{Td}$ scans, whereas, during the treatment period, small lesions were $20 \%$ of the total on Sd and $27 \%$ of the total on Td scans.

\section{Discussion}

This study indicates that the effect on enhancing multiple sclerosis lesions of $44 \mu \mathrm{g}$ of rh-IFN $\beta-1$ a administered once a week is much weaker than with higher doses and more frequent administration ${ }^{5}$; the effect also varies according to the nature and size of the lesions. However, other factors may also contribute to the discrepancy between the results of the present study and those of previous trials using the same or other forms of IFN $\beta-1 \mathrm{a}^{4-7}$ or IFN $\beta-1 b{ }^{1-3}$ Our patient sample was relatively small, and they were followed up for only a short period. Nevertheless, other studies, ${ }^{1-35}$ which were conducted with even smaller sample sizes ${ }^{23}$ or had comparable durations of follow up periods, ${ }^{1-35}$ found a $60 \%-90 \%$ reduction in the number of enhancing lesions. The disparity may have arisen because we selected patients with a lower MRI baseline activity than some of the previous studies, ${ }^{1-3}$ in which there was an inclusion criterion of a mean of at least 0.5 lesions/month over the 6 month baseline period..$^{1-3}$ Pozzilli et $a \bar{l}^{\overline{5}}$ and Paty et $a l^{7}$ although they did not use baseline enhancement as an inclusion criterion, had a mean number of enhancing lesions on the baseline scans which was higher than we had on $\mathrm{Sd}$ scans $(1.8 v 1.2)$. A higher enhancing lesion frequency on the baseline scans would lead to a more pronounced regression to the mean.

The main finding of interest from the present study is that the effect of rh-IFN $\beta-1 \mathrm{a}$ on enhancing multiple sclerosis lesions is not homogeneous, and is greater for small lesions and those seen to enhance after Td Gd. This confirms previous statistical simulations, ${ }^{10}$ which suggested that the higher sensitivity of Td scanning, compared with Sd, for detecting enhancing multiple sclerosis lesions would increase the likelihood of showing a treatment effect, when using the same number of scans. However, a corollary of such an approach is that there may be a risk of picking up an effect that has no clinical relevance. There are indeed several pieces of evidence suggesting that the degree of blood-brain barrier disruption and the severity of the associated damage in the brain parenchyma are mild in $\mathrm{Td}$ lesions. ${ }^{18}$ Small enhancing lesions may also represent areas in which a less severe pathological process is occurring. Thus the present study would suggest that the effect of the drug is inversely related to the degree of blood-brain barrier disruption, in agreement with previous results $^{471920}$ which showed that the effect of the interferons on the frequency of appearance of new enhancing lesions is two to three times greater than on the clinical relapse rate.

The rhIFN $\beta$-1a (REBIF) was supplied to us at no cost by SERONO Pharma (Rome, Italy). The study was also supported by the Associazione Italiana Sclerosi Multipla (AISM). We are grateful to Dr R Garavaglia for her assistance in study management.

1 Stone LA, Frank JA, Albert PS, et al. The effect of interferon- $\beta$ on blood-brain barrier disruptions demonstrated by contrast-enhanced magnetic resonance imaging in relapsing-remitting multiple sclerosis. Ann Neurol in relapsing-remitir

2 Stone LA, Frank JA, Albert PS, et al. Characterization of MRI response to treatment with interferon $\beta-1 \mathrm{~b}$ : ContrastMRI response to treatment with interferon $\beta-1 \mathrm{~b}$ : Contrast-
enhancing MRI lesion frequency as a primary outcome enhancing MRI lesion frequency as
measure. Neurology 1997;49:862-9.

3 Calabresi P, Stone LA, Bash CN, et al. Interferon $\beta$ results in immediate reduction of contrast-enhanced MRI lesions in multiple sclerosis patients followed by weekly MRI. Neurology 1997;48:1446-8.

4 Jacobs DL, Cookfair DL, Rudick RA, et al. Intramuscular interferon $\beta$-1a for disease progression in relapsing multiple sclerosis. Ann Neurol 1996;39:285-94.

5 Pozzilli C, Bastianello S, Koudriavtseva T, et al. Magnetic resonance imaging changes with recombinant human interferon $\beta-1 \mathrm{a}:$ a short term study in relapsing-remitting multiple sclerosis. F Neurol Neurosurg Psychiatry 1996;61: 251-8.

6 Simon JH, Jacobs LD, Campion M, et al. Magnetic resonance studies of intramuscular interferon $\beta-1$ a for relapsing multiple sclerosis. Ann Neurol 1998;43:79-87.

7 Paty DW, Zhao GJ, Hyde R, et al. Comparison between proPaty DW, Zhao GJ, Hyde R, et al. Comparison between pro-
ton density/T2 and gadolinium-enhanced T1 MRI in demton density/T2 and gadolinium-enhanced T1 MRI in dem-
onstrating a treatment effect and dose difference in onstrating a treatment effect and dose difference in
PRISMS trial of Interferon $\beta-1$ a (Rebif) [abstract]. Neurology 1998;50(suppl):A190. 
8 Filippi M, Yousry T, Horsfield MA, et al. A high-resolution three-dimensional gradient echo sequence improves the detection of disease

9 Filippi M, Yousry T, Campi A, et al. Comparison of triple dose versus standard dose gadolinium-DTPA for detection of MRI enhancing lesions in patients with multiple sclerosis. Neurology 1996;46:379-84.

10 Filippi M, Rovaris M, Capra R, et al. A multi-center longitudinal study comparing the sensitivity of monthly MR after standard and triple dose gadolinium-DTPA for monitoring disease activity in multiple sclerosis: implications for phase II clinical trials. Brain 1998;121:2011-20.

11 Filippi M, Rocca MA, Rizzo G, et al. Magnetization transfer ratios in MS lesions enhancing after different doses of gadolinium. Neurology 1998;50:1289-93.

12 Poser CM, Paty DW, Scheinberg L, et al. New diagnostic criteria for multiple sclerosis: guidelines for research protocriteria for multiple sclerosis: guide
cols. Ann Neurol 1983;13:227-31.

13 Lublin FD, Reingold SC, the National Multiple Sclerosis Society (USA) Advisory Committee on Clinical Trials of New Agents in Multiple Sclerosis. Defining the clinical New Agents in Multiple Sclerosis. Defining the clinical course of multiple sclerosis: result
vey. Neurology 1996;46:907-11.

14 Kurtzke JF. Rating neurological impairment in multiple sclerosis: an expanded disability status scale (EDSS). Neurology 1983;33:1444-52.
15 Miller DH, Barkhof F, Berry I, et al. Magnetic resonance imaging in monitoring the treatment of multiple sclerosis: concerted action guidelines. F Neurol Neurosurg Psychiatry 1991;54:683-8.

16 Barkhof F, Filippi $\mathrm{M}$, van Waesberghe JH, et al. Interobserver variation in reporting gadolinium-enhanced MS lesions: consensus rules and effect of training. Neurology 1997;49:1682-8.

17 Rovaris M, Filippi M, Calori G, et al. Intra-observer reproducibility in measuring new putative MR markers of demyelination and axonal loss in multiple sclerosis: a comparison with $\mathrm{T}_{2}$-weighted images. F Neurol 1997;244:266-70.

18 Rovaris M, Mastronardo G, Gasperini C, et al. MRI evolution of new MS lesions enhancing after different doses of gadolinium. Acta Neurol Scand 1998;98:90-3.

19 Paty DW, Li DKB, UBC MS/MRI Study Group, IFNB Multiple Sclerosis Study Group. Interferon $\beta-1 \mathrm{~b}$ is effective in relapsing-remitting multiple sclerosis. II. MRI analysis results of a multicenter, randomized, double-blind, placebo-controlled trial. Neurology 1993;43:662-7.

20 The IFNB Multiple Sclerosis Study Group, the University of British Columbia MS/MRI Analysis Group. Interferon $\beta-1 b$ in the treatment of multiple sclerosis: final outcome of the randomized controlled trial. Neurology 1995;45:127785 